\title{
Three Patients with Dysphagia Following Hospitalization and Respiratory Support for Severe COVID-19 Pneumonia
}

\author{
Layal A Olaywan ${ }^{1 *}$, Ralph M Nehme ${ }^{2 *}$, Nadine M Francis ${ }^{1}$, Ghina Fahd ${ }^{3}$ and Youssef B Haddad ${ }^{4}$ \\ ${ }^{1}$ Department of Pulmonary and Critical Care, Lebanese Hospital Geitaoui, Lebanon \\ ${ }^{2}$ Department of Pulmonary and Critical Care, Lebanese American University Medical Center-Rizk Hospital, Lebanon \\ ${ }^{3}$ Department of Medicine, Lebanese University, Lebanon \\ ${ }^{4}$ Head of Department of Pulmonary and Critical Care, Lebanese Hospital Geitaoui, Lebanon
}

Submission: November 30, 2021; Published: December 13, 2021

*Corresponding author: Ralph M. Nehme, Lebanese American University Medical Center - Rizk Hospital (LAUMCRH), Lebanon

Layal A Olaywan, Lebanese Hospital Geitaoui, Achrafieh, Beirut, Lebanon

\section{Abstract}

Background: During the coronavirus disease 2019 (COVID-19) pandemic, patients with severe pneumonia may require hospitalization and respiratory support. Oropharyngeal dysphagia may occur due to lack of muscle coordination of the respiratory and swallowing mechanisms in acute respiratory distress syndrome (ARDS) or as a consequence of intervention for respiratory support. This report is of a series of three patients who were hospitalized for severe COVID-19 pneumonia who developed dysphagia.

Case Series: Three patients patients were diagnosed with severe COVID-19 pneumonia with positive reverse transcription-polymerase chain reaction (RT-PCR) testing for SARS-CoV-2. Case 1: A 69-year-old man hospitalized with COVID-19 pneumonia and who underwent noninvasive mechanical ventilation followed by difficulty in swallowing. Case 2: An 84-year-old woman hospitalized with COVID-19 pneumonia and developed confusion, disorientation, swallowing difficulties, and aspiration pneumonia. Case 3: An 87-year-old man who developed ARDS following hospital admission with COVID-19 pneumonia.

Conclusion: These cases have shown that dysphagia may develop in patients hospitalized with severe COVID-19 pneumonia, either due to respiratory interventions or due to ARDS, and should be identified and actively managed to prevent further complications due to aspiration of gastric contents.

Keywords: Coronavirus disease 2019; COVID-19; Dysphagia; Pneumonia; Case report

\section{Introduction}

With more than 150 million cases to date and 6.93 million global deaths [1], COVID-19 has massively modified and overwhelmed the structure of the healthcare system worldwide, requiring persistent novel approaches for healthcare delivery $[2,3]$. The most common clinically reported presentation of severe COVID-19 cases is acute respiratory distress syndrome (ARDS) (67\% of COVID-19 patients with the severe illness) [3], rapidly progressing to multi-organ failure and death [4]. Some of these patients require intubation and mechanical ventilation.

However, post-extubation dysphagia is a common concern in intensive care unit patients with prevalence ranging between 3 and $62 \%$ and is significantly associated with increased intubation time [5,6]. Dysphagia can be related to multiple etiologies attributed to cognitive and mechanical changes [7]. Intubation has been shown to cause laryngeal damage in $83 \%$ of cases with $47 \%$ incidence of full-thickness tracheal erosion (FTTE) and tracheoesophageal fistula (TEF) [8] with only a few intubated patients being spared from injury [9]. Additional collateral damage in COVID-19 patients may be attributed to the necessity of rapid intervention in emergency cases and the difficult delivery of care in personal protective equipment [10]. In regard to cognitive etiologies, critical illness is an important predictor of impaired swallowing reflex coordination [7]. This reflex may be further weakened in COVID-19 patients having incoordination between swallowing and respiration, and in whom central and peripheral 


\section{Juniper Online Journal of Case Studies}

nerve involvement is already proven [11-13]. According to recent findings, dysphagia in COVID-19 positive patients was thought to be related to the lack of the respiratory-swallowing coordination seen in ARDS or due to mechanical respiratory support damage [11]. In addition, during this pandemic, there has been several case reports associating COVID-19 infection with Guillain Barre Syndrome, a disease that is manifested by an acute ascending weakness and sometimes dysphagia, and that the weakness was more severe in COVID-19 infected patients necessitating ICU admission. This disease can also contribute to the appearance of dysphagia in some COVID-19 positive patients [14].

Metabolic and nutritional adverse effects of COVID-19 infection may also contribute to the delayed recovery from dysphagia in critically ill patients, which usually holds unfavorable outcomes and late results. Dysphagia and consequently loss of appetite and worse nutritional status as adverse effects of COVID-19 infection may also contribute to negative prognostic factors when underestimated, holding unfavorable outcomes for COVID-19 patients $[15,16]$.

To date, multiple cases of dysphagia following COVID-19 have been reported and were associated with poor outcomes such as aspiration pneumonia, increased length of hospitalization, and prolonged periods of rehabilitation [11]. In light of high associated mortality rates and with little data on appropriate management, the study of dysphagia in COVID-19 patients can contribute to the development of prompt evaluation and intervention procedures done in a multidisciplinary context including speech and language therapy assessment, practice swallow, progressive modifications of diet textures and adaptation to environmental factors to better predict patient recovery and optimize rehabilitation helping them return to their baseline swallow function.

This report is of a series of three patients who were hospitalized for severe COVID-19 pneumonia who developed dysphagia.

\section{Case Series}

We present a series of three cases of dysphagia in COVID-19 patients collected retrospectively from a single university hospital in Beirut. Patients included in the study presented between December 2020 and January 2021. Biographical details are described in Table 1. Pneumonia was classified as critical if the patient required mechanical ventilation and severe if he required noninvasive ventilation. Diagnosis of COVID-19 in each of the three patients was confirmed by two positive nasopharyngeal specimens on RT-PCR collected one week apart. PCR kit Name: Gene finder COVID-19 fast RealAmp kit. Manufacturer: OSANG Healthcare, Korea

Table 1:

\begin{tabular}{|c|c|c|c|}
\hline & Patient 1 & Patient 2 & Patient 3 \\
\hline Sex & Male & Female & Male \\
\hline Age & 69 & 84 & 87 \\
\hline Comorbidities & $\begin{array}{c}\text { HTN, DM, and polymyalgia rheu- } \\
\text { matic }\end{array}$ & HTN, DM, and CAD & HTN, DM, and CKD \\
\hline Signs \& Symptoms on Admission & Dyspnea and oxygen desaturation & $\begin{array}{l}\text { Dyspnea and oxygen desat- } \\
\text { uration }\end{array}$ & Dyspnea, cough, and fever \\
\hline Labs & $\begin{array}{l}\uparrow \mathrm{WBC} \\
\uparrow \mathrm{CRP} \\
\uparrow \mathrm{IL}-6 \\
\uparrow \mathrm{LDH}\end{array}$ & $\begin{array}{l}\text { Neutrophilia } \\
\quad \uparrow \text { CRP } \\
\quad \uparrow \text { IL-6 }\end{array}$ & $\begin{array}{l}\uparrow \mathrm{CRP} \\
\uparrow \mathrm{IL}-6 \\
\uparrow \mathrm{PCT}\end{array}$ \\
\hline Imagery & $\begin{array}{l}\text { Bilateral GGO } 80 \% \text { parenchymal } \\
\text { involvement. Minimal pneumotho- } \\
\text { rax in left upper lobe }\end{array}$ & $\begin{array}{l}\text { Bilateral infiltrates with } \\
\text { moderate bilateral pleural } \\
\text { effusion }\end{array}$ & $\begin{array}{l}\text { Bilateral GGO 55\% parenchymal } \\
\text { involvement with consolidations } \\
\text { and air bronchogram }\end{array}$ \\
\hline Severity of Pneumonia at Admission & Severe & Severe & Critical \\
\hline Oxygen Therapy duration (days) & 48 & 22 & 55 \\
\hline Mechanical Ventilation (days) & 12 (after aspiration) & 0 & 8 \\
\hline Prone Position & No & No & Yes \\
\hline Time from Admission to Outcome (days) & 48 & 22 & 55 \\
\hline Time from Admission to Dysphagia & 20 & 14 & 9 \\
\hline Complications & $\begin{array}{c}\text { Dysphagia Aspiration pneumonia, } \\
\text { cardiac arrest }\end{array}$ & $\begin{array}{c}\text { Dysphagia Aspiration pneu- } \\
\text { monia, myocarditis, cardiac } \\
\text { arrest }\end{array}$ & $\begin{array}{l}\text { Dysphagia GI bleed, aspiration } \\
\text { pneumonia }\end{array}$ \\
\hline
\end{tabular}




\section{Case 1}

A 69-year-old male was admitted to the intensive care unit (ICU) for dyspnea and oxygen desaturation due to severe COVID-19 pneumonia that required several days of noninvasive mechanical ventilation (NIV). After three weeks, even though noninvasive ventilation was gradually tapered to high flow nasal cannula the patient showed some difficulty swallowing food. Assessment using a fibroscopic examination showed normal anatomy with normal movement of vocal cords but sensation was found to be abnormal accompanied by extreme weakness and salivary accumulation in the pharynx with difficulty swallowing it. CT of the brain showed no abnormalities. Patient was switched to parenteral nutrition and he was put nil per os to avoid further aspiration.

A few days later, the patient manifested an increase in oxygen requirement and increased dyspnea. CT of the chest was repeated and showed right upper lobe consolidation and bilateral lower lobes consolidations that are possibly due to aspiration pneumonia. He was started on antibiotics, but he deteriorated clinically, got intubated and eventually died seven weeks after admission.

\section{Case 2}

An 84-year-old female was admitted to regular floor for dyspnea and oxygen desaturation due to severe pneumonia caused by COVID-19 infection. She required several days of 15L/ minute oxygen delivery by nonrebreather face mask, she was not mechanically ventilated.

Two weeks later, the patient had worsening dyspnea and an increase in oxygen requirements. Transthoracic echocardiography showed type I diastolic dysfunction with an ejection fraction (EF) of $45 \%$ and a systolic pulmonary artery pressure (sPAP) of $40 \mathrm{mmHg}$. Coronarography was done and showed no coronary artery stenosis. Her condition was further complicated by confusion, disorientation, swallowing difficulties, and aspiration pneumonia. Brain MRI showed atrophy with no acute infarcts or ischemic changes. The patient required a gastrostomy tube for feeding. But the patient deteriorated and death occurred three weeks after admission.

\section{Case 3}

An 87-year-old male was admitted to the ICU for confirmed severe COVID-19 pneumonia with dyspnea, cough, and fever. The patient required oxygen supplementation with a high-flow nasal cannula then got intubated for ARDS. His condition was complicated by a gastrointestinal bleed requiring multiple units of blood transfusion.

One week later, the patient showed recovery, was extubated to a nasal cannula of 3/L min oxygen and was transferred to the regular floor. On the twelfth day after admission, the patient complained of dysphagia, he developed fever and cough with expectoration, and had an increase in oxygen requirements. Chest X-ray showed new left-sided pneumonia requiring therapeutic bronchoscopy and removal of mucus plugs. An otorhinolaryngologist was consulted for the assessment of oropharyngeal dysphagia. GAG reflex was absent bilaterally. Fibroscopy was done which showed negative epiglottic tactile sense, laryngeal reflex, or swallowing reflex. A jejunostomy tube was then inserted, and the patient was discharged from the hospital on enteral nutrition, with persistent dysphagia eight weeks after admission (Table 1).

\section{Discussion}

Swallowing is a sequential and coordinated developmental behavior connecting more than thirty muscles and six cranial nerves $[17,18]$. If disrupted, a wide range of symptoms may occur resulting in adverse patient outcomes, such as malnutrition, aspiration pneumonia, prolonged hospitalization, and death $[6,19]$. Most people with dysphagia also report a substantial decrease in their quality of life influencing their eating behaviors and psychological well-being [20].

The studied patients were elderly with various comorbidities such as diabetes mellitus, hypertension, and inflammatory diseases which explains the severity of their COVID-19 condition and the necessity of intensive care unit admission [21]. Dysphagia in ICU patients has been previously related to multiple etiologies, ranging from traumatic upper airway injury to neurological abnormalities [7].

In fact, dysphagia is a common symptom that accompanies a number of neurologic disorders since it needs meticulous neural coordination within a network of interneurons located in the brainstem, hence called neurogenic dysphagia [22]. In fact, the first patient demonstrated abnormal sensation and prominent weakness, which are two important mechanisms in the development of ICU dysphagia. As suggested by Zuercher et al. [6] \& Brodsky et al. [23] specific swallow-related muscular weakness and reduced local sensation are key components of dysphagia and important predictors of aspiration pneumonia, hence the exacerbated condition of the patient $[6,23]$. It is important to note that all patients had elevated IL-6; this can reflect a level of neuroinflammation and blood-brain barrier dysfunction in patients with COVID-19, supporting the hypothesis of neurogenic dysphagia [24]. These findings go along with the neurologic deficits correlated with both ARDS and ICU practices that are necessary in the treatment of COVID-19 patients [11].

Our second patient also showed symptoms of dysphagia of neurologic origin that could not be explained by a stroke event which is the most common cause of neurogenic dysphagia [25]. This hypothesis is further strengthened by associated findings of delirium, another known cause of dysphagia, and MRI results showing acquired brain injury, probably due to lack of oxygen in this patient. In fact, studies suggest that delirium is an important predictor of dysphagia and aspiration in hospitalized elderly patients [26]. Hence, we can conclude that there might be several 
mechanisms responsible for dysphagia in one patient, especially the elderly ones.

All findings can also be explained by the increasing evidence of central nervous system implication in COVID-19, notably brain stem involvement $[27,28]$. These effects arise from the established direct neurotrophic effect of the virus or by a post-infectious autoimmune mechanism [29]. Interestingly, Matschke et al. [30] postulated that cranial nerve involvement may be associated with symptoms of dysphagia found in COVID-19 patients, implying glossopharyngeal and vagal effect on bulbar muscle dysfunction resulting in dysphagia, although brainstem neuropathological findings were not found in the post-mortem period [30]. Moreover, the various multisystemic effects of COVID-19 on elderly ICU patients may favor a decompensated state hence the development of critical illness polyneuropathy with accompanied dysphagia [31].

Aside from the neurological background, the risk of dysphagia is further increased in patients who require mechanical intubation and/or tracheostomies. Post-extubation dysphagia is more likely to occur with prolonged intubation time, but can still occur with a few days of intubation [32]. A higher incidence of dysphagia was reported when intubation was upon emergency admission, which leaves room for more laryngeal trauma upon insertion of the tube, especially in COVID-19 patients in whom prudent techniques should be adopted [33]. This can explain the development of aspiration in the third patient, in whom aspiration pneumonia occurred due to dysphagia and exacerbated his condition. This hypothesis is further reinforced by the concomitant presence of a neurologic etiology in this patient explained by the abnormal reflexes. Similar mechanisms explain the development of dysphagia in the fourth patient, in whom the need for reintubation and rapid decompensation led to death. In fact, aspiration pneumonia endotracheal obstruction and impaired mucus clearance may be exacerbated by prone-position of mechanically ventilated, a therapeutic intervention that is widely used in COVID-19 patients used to optimize oxygenation leading to atelectasis and pneumonia [11]. In addition to that, critically-ill patients are strong candidates for myopathy and polyneuropathy, further complicating their illness, compromising their rehabilitation, and prolonging their ICU stay $[34,35]$.

Bacterial co-infections need to be taken into consideration since intensive care unit patients are at a greater risk for nosocomial infections and these infections are major predictors of severity and poor outcome in COVID-19 patients [36,37]. These findings are similar to those in Dziewas et al. [37] in which patients with dysphagia frequently had superimposed bacterial infections causing additional major pulmonary complications [37].

This highlights the importance of thorough diagnostic and management procedures starting from clinical screening protocol as bedside assessment which is less costing and time consuming to video fluoroscopic assessment of swallowing when available because of the need to transfer the patient to the radiological department to predict and anticipate treatment of oropharyngeal dysphagia because once set, the progression to aspiration pneumonia is "silent" and life-threatening [38]. A Taking into account that the diagnosis of dysphagia in COVID-19 patients is not always obvious and gold-standard diagnostic methods can't be relied on, familiarity and attention should be improved among physicians [39].

Various treatment modalities of dysphagia in COVID-19 patients have been explored and showed interesting results. A recent case study reported a favorable restoration of safe swallowing function in a patient with COVID-19 post-extubation neurogenic dysphagia using pharyngeal electrical stimulation, a method formerly used for dysphagia of stroke origin [40]. This method can be used to accelerate recovery and reduce total hospital stay. The use of speech and language therapy (SLT) also showed favorable results in rehabilitation of COVID-19 patients with dysphagia and dysphonia after ICU stay [41].

\section{Conclusion}

The main finding in this case series is that dysphagia is putting COVID patients at higher risk of airway compromise. Dysphagia may develop in patients hospitalized with severe COVID-19 pneumonia, either due to respiratory interventions or due to ARDS, and should be identified and actively managed to prevent further complications due to aspiration of gastric contents. This complication is responsible for prolonged hospitalization and even poor overall outcome. There is still a need for large-scale prospective studies to determine optimal early diagnostic and management procedures in these patients. In the absence of established standard guidelines, the key element is the need for optimization of systematic dysphagia screening in ICU COVID-19 patients and targeted swallow rehabilitation methods. Due to the limited information currently available, the concomitant presence of both pathologies remains a major challenge for ICU physicians.

\section{References}

1. Dyer 0 (2021) Covid-19: Study claims real global deaths are twice official figures. BMJ 373: n1188.

2. Miller IF, Becker AD, Grenfell BT, Metcalf CJE (2020) Disease and healthcare burden of COVID-19 in the United States. Nat Med 26(8): 1212-1217.

3. Armocida B, Formenti B, Ussai S, Palestra F, Missoni E (2020) The Italian health system and the COVID-19 challenge. Lancet Public Health 5(5): e253.

4. Wu C, Chen X, Cai Y, Xia J, Zhou X, et al. (2020) Risk Factors Associated with Acute Respiratory Distress Syndrome and Death in Patients with Coronavirus Disease 2019 Pneumonia in Wuhan, China. JAMA Intern Med 180(7): 934-943.

5. Skoretz SA, Flowers HL, Martino R (2010) The incidence of dysphagia following endotracheal intubation: a systematic review. Chest 137(3): 665-673. 
6. Zürcher P, Moret C, Dziewas R, Schefold J (2019) Dysphagia in the intensive care unit: Epidemiology, mechanisms, and clinical management. Crit Care 1: 23.

7. Kwok AM, Davis JW, Cagle KM, Sue LP, Kaups KL (2013) Post-extubation dysphagia in trauma patients: it's hard to swallow. Am J Surg 206(6): 924-927.

8. Balakrishnan K, Brenner MJ, Hillel AT (2021) Laryngeal and Tracheal Pressure Injuries in Patients With COVID-19. JAMA Otolaryngol Head Neck Surg 147(5): 484-485.

9. Colton House J, Noordzij JP, Murgia B, Langmore S (2011) Laryngeal injury from prolonged intubation: a prospective analysis of contributing factors. The Laryngoscope 121(3): 596-600.

10. Shrestha GS, Shrestha N, Lamsal R, Pradhan S, Shrestha A, et al. (2021) Emergency Intubation in Covid-19. N Engl J Med 384(7): e20.

11. Mohan R, Mohapatra B (2020) Shedding Light on Dysphagia Associated With COVID-19: The What and Why. OTO Open 4(2).

12. Doblan A, Kaplama ME, Ak S, Basmaci N, Tarini EZ, et al. (2021) Cranial nerve involvement in COVID-19. Am J Otolaryngol 42(5): 102999.

13. Gao J, Zhou C, Zhang H (2020) Mechanical ventilation in patients with acute ischemic stroke: from pathophysiology to clinical practice. Crit Care 24(1): 139.

14. Koike H, Chiba A, Katsuno M (2021) Emerging Infection, Vaccination, and Guillain-Barré Syndrome: A Review. Neurol Ther 10(2): 523-537.

15. Brodsky MB, Huang M, Shanholtz C, Mendez-Tellez PA, Palmer JB, et al. (2017) Recovery from Dysphagia Symptoms after Oral Endotracheal Intubation in Acute Respiratory Distress Syndrome Survivors. A 5-Year Longitudinal Study. Ann Am Thorac Soc 14(3): 376-383.

16. Singer P (2021) Nutritional and metabolic management of COVID-19 intensive care patients. J Intensive Med 1(1): 31-34.

17. Frajkova Z, Tedla M, Tedlova E, Suchankova M, Geneid A (2020) Postintubation Dysphagia During COVID-19 Outbreak-Contemporary Review. Dysphagia 35(4): 549-557.

18. Costa MMB (2018) Neural Control of Swallowing. Arq Gastroenterol 55Suppl 1(Suppl 1): 61-75.

19. Garcia JM, Chambers E (2010) Managing dysphagia through diet modifications. Am J Nurs 110(11): 26-33.

20. Ekberg O, Hamdy S, Woisard V, Wuttge-Hannig A, Ortega P (2002) Social and psychological burden of dysphagia: its impact on diagnosis and treatment. Dysphagia 17(2): 139-146.

21. Fang X, Li S, Yu H, Wang P, Zhang Y, et al. (2020) Epidemiological, comorbidity factors with severity and prognosis of COVID-19: a systematic review and meta-analysis. Aging 12(13): 12493-12503.

22. Sawczuk A, Mosier KM (2001) Neural control of tongue movement with respect to respiration and swallowing. Crit Rev Oral Biol Med Off Publ Am Assoc Oral Biol 12(1): 18-37.

23. Brodsky MB, De I, Chilukuri K, Huang M, Palmer JB (2018) Coordination of Pharyngeal and Laryngeal Swallowing Events During Single Liquid Swallows After Oral Endotracheal Intubation for Patients with Acute Respiratory Distress Syndrome. Dysphagia 33(6): 768-777.

24. Achar A, Ghosh C (2020) COVID-19-Associated Neurological Disorders: The Potential Route of CNS Invasion and Blood-Brain Relevance. Cells 9(11): E2360.
25. Buchholz DW (1994) Dysphagia associated with neurological disorders. Acta Otorhinolaryngol Belg 48(2): 143-155.

26. Sasegbon A, Dark P, O Shea L, Hamdy S (2016) PTU-119 Association Between Acute Sepsis and Oropharyngeal Dysphagia in A Hospitalised Elderly Population. Gut 65: A114-A115.

27. von Weyhern CH, Kaufmann I, Neff F, Kremer M (2020) Early evidence of pronounced brain involvement in fatal COVID-19 outcomes. Lancet Lond Engl 395(10241): e109.

28. Manganelli F, Vargas M, Iovino A, Iacovazzo C, Santoro L, et al. (2020) Brainstem involvement and respiratory failure in COVID-19. Neurol Sci 47(1): 1663-1665.

29. Najjar S, Najjar A, Chong DJ, Pramanik BK, Kirsch C, et al. (2020) Central nervous system complications associated with SARS-CoV-2 infection: integrative concepts of pathophysiology and case reports. J Neuroinflammation 17(1): 231.

30. Matschke J, Luetgehetmann M, Hagel C, Sperhake P, Schröder A, et al. (2020) Neuropathology of patients with COVID-19 in Germany: a postmortem case series. Lancet Neurol.

31. Ponfick M, Linden R, Nowak DA (2015) Dysphagia--a common, transient symptom in critical illness polyneuropathy: a fiberoptic endoscopic evaluation of swallowing study. Crit Care Med 43(2): 365-372.

32. Kim MJ, Park YH, Park YS, Song YH (2015) Associations Between Prolonged Intubation and Developing Post-extubation Dysphagia and Aspiration Pneumonia in Non-neurologic Critically Ill Patients. Ann Rehabil Med 39(5): 763-771.

33. Schefold JC, Berger D, Zürcher P, Lensch M, Perren A, et al. (2017) Dysphagia in Mechanically Ventilated ICU Patients (DYnAMICS): A Prospective Observational Trial. Crit Care Med 45(12): 2061-2069.

34. Hund E (1999) Myopathy in critically ill patients. Crit Care Med 27(11): 2544-2547.

35. Zhou C, Wu L, Ni F, Ji W, Wu J, et al. (2014) Critical illness polyneuropathy and myopathy: a systematic review. Neural Regen Res 9(1): 101-110.

36. Zhu X, Ge Y, Wu T, Zhao K, Chen Y, et al. (2020) Co-infection with respiratory pathogens among COVID-2019 cases. Virus Res 285: 198005.

37. Dziewas R, Hufelschulte LM, Lepper J, Sackarnd J, Minnerup J, et al. (2021) Dysphagia in Patients with Severe Coronavirus Disease 2019-Potential Neurologic Etiologies. Crit Care Explor 3(1): e0332.

38. Sassi FC, Medeiros GC, Zilberstein B, Jayanthi SK, de Andrade CRF (2017) Screening protocol for dysphagia in adults: comparison with videofluoroscopic findings. Clinics 72(12): 718-722.

39. Longo L, Amitrano A, De Vincentiis M, Greco A, Ruoppolo G (2021) The Bedside Clinical Examination as a key element of the swallowing assessment during the COVID 19 Pandemic. Eur Ann Otorhinolaryngol Head Neck Dis 138(4): 313.

40. Traugott M, Höpler W, Kitzberger R, Seitz T, Baumgartner S, et al. (2020) Successful treatment of intubation-induced severe neurogenic post-extubation dysphagia using Pharyngeal Electrical Stimulation in a COVID-19 survivor: a case report. Journal of Medical Case Reports 15(148).

41. RCSLT-COVID-19-SLT-rehab-pathway_15-July-2020_FINAL.pdf 
(C) This work is licensed under Creative BY DOI: 10.19080/JOJCS.2021.13.555851
Your next submission with Juniper Publishers will reach you the below assets

- Quality Editorial service

- Swift Peer Review

- Reprints availability

- E-prints Service

- Manuscript Podcast for convenient understanding

- Global attainment for your research

- Manuscript accessibility in different formats

(Pdf, E-pub, Full Text, Audio)

- Unceasing customer service

Track the below URL for one-step submission https://juniperpublishers.com/online-submission.php 